\title{
HOPF BIFURCATION IN QUASI-GEOSTROPHIC CHANNEL FLOW
}

\author{
ZHI-MIN CHEN*, MICHAEL GHIL ${ }^{\dagger}$, AND SHOUHONG WANG $\ddagger$
}

\author{
This PAPER IS DEDICATED TO THE MEMORY OF JACQUes-Louis LiONS
}

\begin{abstract}
In this article, we conduct a rigorous stability and bifurcation analysis for a highly idealized model of planetary-scale, atmospheric and oceanic flows. The model is governed by the twodimensional, quasi-geostrophic equation for the conservation of vorticity in an East-West oriented, periodic channel. The main result is the existence of Hopf bifurcation of the flow as the Reynolds number crosses a critical value.
\end{abstract}

Key words. Quasi-geostrophic channel flow, Hopf bifurcation

AMS subject classifications. 35Q30, 86A05, 86A10, 76E20, 58C40

1. Introduction. A key problem in the study of climate dynamics is to understand and predict the periodic, quasi-periodic, aperiodic, and fully turbulent characteristics of large-scale atmospheric and oceanic flows. Bifurcation theory enables one to determine how qualitatively different flow regimes appear and disappear as control parameters vary; it provides us, therefore, with an important method to explore the theoretical limits of predicting these flow regimes. In the present paper, we study bifurcations of the original partial differential equations (PDEs) that govern geophysical flows, rather than the truncated systems of ordinary differential equations (ODEs) that are used most often in such studies. This should allow us to overcome some of the limitations of the numerical bifurcation results that dominate the climate dynamics literature up to this point, and to capture the essential dynamics of the governing PDE systems.

The basic equations of the large-scale atmospheric and oceanic circulation are the primitive equations. These equations can be derived from the full Navier-Stokes equations with gravity, rotation, and variable density by neglecting vertical accelerations (the so-called hydrostatic approximation) and compressibility effects (i.e., sound waves); see Ghil and Childress [8], Kalnay [15], Lions, Temam and Wang [17, 18], and Pedlosky [28]. One philosophy in the geosciences is to study in great detail simplified models that approximate well the dominant balance of forces on the planetary-scale, atmospheric and oceanic flows before addressing the more complete PDE systems that govern these flows in all their complexity. By starting with models that incorporate only the most important effects, and by gradually bringing in others, one is able to proceed inductively and avoid thereby the pitfalls inevitably encountered when a great many poorly understood factors are introduced all at once.

* Department of Ship Science, University of Southampton, Southampton SO17 1BJ, UK and Nankai Institute of Mathematics, Nankai University, Tianjin 300071, People's Republic of China (zhimin@ship.soton.ac.uk). This author was supported in part by the National Natural Science Foundation of China.

$\dagger$ Department of Atmospheric Sciences and Institute of Geophysics and Planetary Physics, University of California, Los Angeles, CA 90095 (ghil@atmos.ucla.edu, http://www.atmos.ucla.edu/tcd). This author was supported in part by the National Science Foundation under Grant ATM-0082131.

${ }^{\ddagger}$ Department of Mathematics, Indiana University, Bloomington, IN 47405 (showang@indiana.edu, http://www.indiana.edu/ fluid). This author was supported in part by the Office of Naval Research under Grant N00014-96-1-0425 and by the National Science Foundation under Grant DMS0072612 . 
The ideas of dynamical systems theory and nonlinear functional analysis have been applied so far to climate dynamics mainly by careful numerical studies. These were pioneered by Lorenz [19, 20], Stommel [35], and Veronis [36, 37], among others, who explored the bifurcation structure of low-order models of atmospheric and oceanic flows. More recently, pseudo-arclength continuation methods have been applied to atmospheric (Legras and Ghil [16]) and oceanic (Speich et al. [34] and Dijkstra [7]) models with increasing horizontal resolution. These numerical bifurcation studies have produced so far fairly reliable results for two classes of geophysical flows: (i) atmospheric flows in a periodic mid-latitude channel, in the presence of bottom topography and a forcing jet; and (ii) oceanic flows in a rectangular mid-latitude basin, subject to wind stress on its upper surface. In both cases, the symmetry properties of the forcing have a decisive effect on the bifurcations that arise - saddle-node (Charney and DeVore [4] and Pedlosky [27]) or Hopf (Legras and Ghil [16] and Jin and Ghil [13]) in the atmospheric channel and pitchfork or Hopf $[1,2,9,12,24,34]$ in the oceanic basin.

More recently, the role of global bifurcations, via homoclinic and heteroclinic orbits, has been demonstrated numerically in the wind-driven ocean circulation problem, for both shallow-water (Chang et al. [3], Simonnet et al. [32, 33]) and quasigeostrophic (Meacham [23] and Nadiga and Luce [26]) models. Still, the only rigorous proof of a bifurcation in either the atmospheric or the oceanic problem outlined here appears, as far as we know, in the work of Wolansky [39, 40] and it extends solely to the existence of asymmetric stationary solutions.

The present paper addresses the somewhat more difficult problem of proving the existence of Hopf bifurcation in a quasi-geostrophic (QG) flow in two dimensions (2D). The main difficulty in solving this problem is to estimate the crucial information on the spectrum of the linearized problem around the basic flow. The main objective of this article is to overcome this difficulty and bring a new set of tools to the rigorous study of successive bifurcations in geophysical fluid dynamics problems.

More precisely, we conduct a bifurcation analysis of the following idealized, 2-D QG flow problem. The governing equation dictates the conservation of vorticity, as modified by forcing and dissipation:

$$
\partial_{t} \Delta \psi+\varepsilon J(\psi, \Delta \psi)+\partial_{x} \psi=E \triangle^{2} \psi-\tau_{0} \sin \pi y,
$$

where $\psi=\psi(x, y, t)$ is a streamfunction, and $J(\psi, \phi)=\partial_{x} \psi \partial_{y} \phi-\partial_{y} \psi \partial_{x} \phi$ is the advection operator. The $x$-axis is directed to the east and the $y$-axis to the north. The zonal and meridional velocity components $u$ and $v$ are obtained from the streamfunction by

$$
u=-\psi_{y}, \quad v=\psi_{x} .
$$

The relative vorticity $\xi$ and the streamfunction $\psi$ are related by the Poisson equation

$$
\triangle \psi=\xi
$$

Equation (1.1) is derived from either the shallow-water equations with rotation or the primitive equations by the so-called quasi-geostrophic (QG) approximation, which assumes that the balance between the Coriolis force and the pressure gradient dominates the flow. This approximation corresponds to a singular perturbation that filters out the Poincaré waves, also called inertia-gravity waves (i.e., gravity waves modified by the presence of rotation). The QG equation (1.1) only supports Rossby 
waves, whose phase velocity - in the absence of forcing and dissipation, i.e., with a zero right-hand side - is comparable to the characteristic particle velocity; see Ghil and Childress [8], and Pedlosky [28]. Wolansky [40] studied the so-called barotropic, 2-D version of the QG model (1.1), while Wang [38] obtained results on existence, uniqueness, and long-time dynamics of the so-called baroclinic, 3 -D version.

The flow domain is a rectangular region $\Omega=\{(x, y) ; 0 \leq x \leq 2 / a ; 0 \leq y \leq 2\}$. For simplicity, we use here only the zonal component of the forcing. In an atmospheric model, this forcing represents - in the QG vorticity equation (1.1), in which there are no explicit thermodynamic effects - the transfer of angular momentum into midlatitudes due to the tropical Hadley cell (Lorenz [21]). Alternatively, one can think about a zonal forcing jet that would be in perfect geostrophic equilibrium with the pole-to-equator temperature gradient (Lorenz [20] and Ghil and Childress [8]).

In an oceanic model, the time-and-longitude independent forcing on the righthand side of (1.1) is the curl of the wind stress

$$
\nabla \times \tau=-\tau_{0} \sin \pi y
$$

a wind stress of the form $\tau=-\tau_{0}(\cos \pi y, 0)$ mimics the annually averaged zonal wind distribution over the North Atlantic or North Pacific, with westerly (i.e., eastward) winds over the mid-latitudes and easterlies in the tropics and polar latitudes.

The parameters $\varepsilon$ and $E$ are positive constants, called the Rossby and Ekman numbers, respectively. They measure the relative importance of nonlinearity and lateral diffusion. The effect of the bottom friction is neglected in this article. The Reynolds number is defined here as

$$
R=\frac{\varepsilon}{E} .
$$

The unknown streamfunction $\psi$ satisfies periodic boundary conditions at $x=$ $0,2 / a$ and free-slip boundary conditions at $y=0,2$ :

$$
\left\{\begin{array}{l}
\psi(t, 2 / a, y)=\psi(t, 0, y), \\
\psi(t, x, 0)=\psi(t, x, 2)=0 ; \partial_{y}^{2} \psi(t, x, 0)=\partial_{y}^{2} \psi(t, x, 2)=0 .
\end{array}\right.
$$

It is readily seen that (1.1) with (1.2) admits the steady-state solution $\psi_{0}=\psi_{0}(y)$, where

$$
\psi_{0}=\frac{\tau_{0}}{\pi^{4} E} \sin \pi y .
$$

We shall take $\tau_{0}=1$ below for simplicity.

This mid-latitude channel with zonal periodicity is a better model for the atmospheric problem that we outlined above than for the oceanic one. Still, the methods we apply might eventually be extended to the latter. As mentioned already, Wolansky [39] studied existence, uniqueness and stability of stationary solutions of (1.1) in the presence of topography and free-surface effects, which are omitted here. Wolansky [40] showed, for a domain bounded by a closed streamline and non-divergent forcing, that sufficient conditions exist under which a branch of asymmetric stationary solutions bifurcates from the symmetric branch obtained when the domain, as well as the forcing, admits a symmetry group. His results were shown to apply in an infinite channel with the symmetry group of zonal translations.

The main objective of this article is to prove the following theorem.

THEOREM 1.1. 
(i) Let $a \geq \sqrt{3} / 2$ and $E>0$. Then (1.1) and (1.2) are linearly stable around $\psi_{0}$ for any Reynolds number $R>0$, where $\psi_{0}=\psi_{0}(y)$ is given by (1.3).

(ii) Let $1 / 2 \leq a \leq 3 / 4$ and $E>0$. Then there exists a critical Reynolds number $R_{0}>0$ for (1.1) and (1.2). Moreover (1.1) and (1.2) admit a nontrivial, time-periodic, classical solution $\psi_{R}$ branching off $\psi_{0}$ as the Reynolds number $R$ crosses $R_{0}$, provided that $E>c_{0}$ for some constant $c_{0}>0$.

This theorem is based essentially on the eigenvalue analysis of the spectral problem with respect to the linearization of (1.1) and (1.2) around $\psi_{0}$, by using the continued-fraction method first introduced by Meshalkin and Sinai [25]. For the 2D Navier-Stokes equations, without the Coriolis term and with periodic boundary conditions in both the $x$ and $y$ directions, stability and bifurcation were studied in $[25,11,5]$. For the 3-D Navier-Stokes equations, without the Coriolis term and with periodic boundary conditions in three directions, pitchfork bifurcation was studied by Chen and Wang [6]. They showed that the bifurcated branches exist for all Reynolds number values past the critical one and that the stationary solutions on these branches stay bounded as $R \rightarrow \infty$.

Meshalkin and Sinai [25] deduced the linear stability of the steady state (1.3) when $a=1$ with respect to all Reynolds number, Iudovich [11] proved the existence of steady-state bifurcation when $0<a<1$, and Chen and Price [5] obtained the existence of Hopf bifurcation for some $a$ with $0<a<\sqrt{3} / 2$. Steady-state bifurcation, however, no longer occurs for any $a$ under the free-slip boundary condition described by (1.2).

In the present paper, the constant $a$ is bounded from below by $1 / 2$. In fact, by applying our approach here in a more sophisticated manner, the existence of timeperiodic solutions can also be obtained when $0<a<1 / 2$; this will be reported elsewhere. The occurrence of the so-called $\beta$-term $\partial_{x} \psi$ in (1.1), which arises due to the meridional gradient of the planetary vorticity, renders the proof of the transversal crossing condition more difficult. In order to prove the validity of this condition, we have to assume that $E>c_{0}$ for some constant $c_{0}$, although numerical experiments reveal the occurrence of Hopf bifurcation for large $\tau_{0}$, but small $E[12,34,9,32,33]$. Technical difficulties prevent us from covering in Theorem 1.1 the range of $x$-periods given by $3 / 4<a<\sqrt{3} / 2$.

To prove Theorem 1.1, we decompose the streamfunction into a stationary part and a perturbation with exponential time dependence

$$
\psi(x, y, t)=e^{\rho t} \phi(x, y),
$$

where $\phi$ satisfies the boundary condition (1.2). Introducing this solution into the governing equation and linearizing it with respect to the perturbation, we obtain the spectral problem

$$
\rho \Delta \phi+\varepsilon\left(\partial_{x} \phi \partial_{y} \Delta \psi_{0}-\partial_{y} \psi_{0} \partial_{x} \Delta \phi\right)+\partial_{x} \phi=E \Delta^{2} \phi .
$$

Substituting the basic solution $\psi_{0}$ from (1.3) yields

$$
L(\rho) \phi \doteq\left(E \Delta^{2}-\partial_{x}+\frac{R}{\pi^{3}} \cos \pi y\left(\pi^{2}+\Delta\right) \partial_{x}-\rho \Delta\right) \phi=0 .
$$

The stability assertion (i) in the main theorem, i.e. the nonexistence of an eigenvalue $\rho$ with $\operatorname{Re} \rho \geq 0$ for all $R>0$, will be obtained by using the argument that Meshalkin and Sinai [25] first applied to the linear stability analysis of the 2-D NavierStokes equations. 
The main effort is devoted to the proof of the bifurcation assertion (ii) of Theorem 1.1. Using the functional analysis framework of the Hopf bifurcation theorem in an infinite-dimensional setting (Joseph and Sattinger [14], and Marsden and McCracken [22]), Assertion (ii) of this theorem will follow if the following assertions can be shown to hold:

(a) There exists a critical Reynolds number $R_{0}>0$ and an eigenvalue $\rho=\rho\left(R_{0}\right)$ of (1.2) and (1.5) such that $\operatorname{Re} \rho\left(R_{0}\right)=0$ and $\operatorname{Im} \rho\left(R_{0}\right) \neq 0$;

(b) this eigenvalue is simple, i.e.,

$$
1=\operatorname{dim} \bigcup_{n \geq 1}\left\{\phi \in H^{4} ;\left(E \Delta^{2}-\partial_{x}+\frac{R_{0}}{\pi^{3}} \cos \pi y\left(\pi^{2}+\Delta\right) \partial_{x}-\rho \Delta\right)^{n} \phi=0\right\} ;
$$

(c) the transversal crossing condition

$$
\operatorname{Re} \frac{d \rho\left(R_{0}\right)}{d R}>0 .
$$

Here $H^{4}$ denotes the complex space

$$
H^{4}=\left\{\phi \in L_{2}(\Omega) ; \Delta^{2} \phi \in L_{2}(\Omega), \phi \text { satisfies }(1.2)\right\},
$$

which contains the solution space of (1.1) and (1.2). This is a Hilbert space in the norm

$$
\|\phi\|_{H^{4}}=\left\|\Delta^{2} \phi\right\|_{L_{2}(\Omega)} .
$$

It is readily seen from the definition of $H^{4}$ that the bifurcating solution can be represented in the form of the Fourier expansion

$\psi(x, y, t)=\sum_{m=-\infty}^{\infty} \sum_{n \geq 1-k} \sum_{k=0}^{1}\left(X_{m, n, k}(t) \cos \operatorname{ma} x x+Y_{m, n, k}(t) \sin \operatorname{ma} x x\right) \sin (n+k / 2) \pi y$.

This paper is organized as follows. Section 2 contains the proof of Assertion (i) and a basic lemma on formulating the spectral problem. The proof of Assertion (ii) is completed by combining Section 3 on the existence of $R_{0}$ and $\rho\left(R_{0}\right)$, Section 4 on the simplicity of the eigenvalue $\rho=\rho\left(R_{0}\right)$, and Section 5 on the eigenvalue transversal crossing condition. Brief remarks on the role of symmetry breaking in the bifurcation of time-periodic vs. stationary solutions follow in Section 6 .

2. Stability and Equivalent Formulation of the Spectral Problem. Note that the free-slip boundary condition in (1.2) is equivalent to the condition $\phi=\Delta \phi=$ 0 at $y=0$ and $y=2$. An application of this condition to (1.5) yields the generalized boundary condition

$$
\Delta^{n} \phi=0, \text { at } y=0 \text { and } y=2 \quad(n=0,1,2, \ldots) .
$$

Thus the expansion of the unknown function $\phi \in H^{4}$ for the spectral problem represented by (1.2) and (1.5) takes the form

(2.1) $\phi=e^{i m a \pi x} \sum_{n \geq 1-k} i^{n} \phi_{n} \sin (n+k / 2) \pi y, \sum_{n \geq 1-k} n^{4}\left|\phi_{n}\right|^{2}<\infty, k=0,1, i=\sqrt{-1}$. 
The fact that $i^{n}$ appears explicitly in the coefficients of this expansion is only for convenience of notation in the derivation.

For simplicity, we consider only the case $m=1$ in this section. As we shall see, it is important to realize that $k=0$ corresponds to a function $\phi_{k}(x, y)$ that is antisymmetric about the axis $y=1$ of the channel, while $\phi_{k}(x, y)$ is symmetric about this axis for $k=1$.

To prove the linear stability result of Theorem 1.1 and to obtain an equivalent formulation of the spectral problem, we follow the argument of Meshalkin and Sinai [25] by changing the spectral problem to a difference equation. The spectral problem (1.5) with $\phi=\phi(x, y)$ in the form of $(2.1)$ and $m=1$ becomes

$$
\begin{aligned}
& \sum_{n \geq 1-k} 2\left\{\pi^{2}\left[a^{2}+(n+k / 2)^{2}\right] \rho-i a \pi+E \pi^{4}\left[a^{2}+(n+k / 2)^{2}\right]^{2}\right\} i^{n} \phi_{n} \sin (n+k / 2) \pi y \\
= & 2 R \sum_{n \geq 1-k} a\left[a^{2}+(n+k / 2)^{2}-1\right] i^{n+1} \phi_{n} \cos \pi y \sin (n+k / 2) \pi y \\
= & R \sum_{n \geq 1-k} a\left[a^{2}+(n+k / 2)^{2}-1\right] i^{n+1} \phi_{n}[\sin (n+1+k / 2) \pi y+\sin (n-1+k / 2) \pi y] \\
= & R \sum_{n \geq 2-k} a\left[a^{2}+(n-1+k / 2)^{2}-1\right] i^{n} \phi_{n-1} \sin (n+k / 2) \pi y \\
& -R \sum_{n \geq 0} a\left[a^{2}+(n+1+k / 2)^{2}-1\right] i^{n} \phi_{n+1} \sin (n+k / 2) \pi y \\
& -R a\left(a^{2}-3 / 4\right) i \phi_{0} \sin (k / 2) \pi y .
\end{aligned}
$$

Letting

$$
\xi_{n}=a\left(a^{2}+(n+k / 2)^{2}-1\right) \phi_{n},
$$

we have, for $k=1$,

$$
\begin{cases}\frac{2\left\{\pi\left[a^{2}+(n+1 / 2)^{2}\right] \rho-i a+E \pi^{3}\left[a^{2}+(n+1 / 2)^{2}\right]^{2}\right\}}{\operatorname{Ra} \pi\left[a^{2}+(n+1 / 2)^{2}-1\right]} \xi_{n}=\xi_{n-1}-\xi_{n+1}, & n \geq 1, \\ \frac{2\left[\pi\left(a^{2}+1 / 4\right) \rho-i a+E \pi^{3}\left(a^{2}+1 / 4\right)^{2}\right]}{\operatorname{Ra}\left(a^{2}-3 / 4\right)} \xi_{0}+i \xi_{0}+\xi_{1}=0, & n=0,\end{cases}
$$

and, for $k=0$,

$$
\begin{cases}\frac{2\left[\pi^{2}\left(a^{2}+n^{2}\right) \rho-i a \pi+E \pi^{4}\left(a^{2}+n^{2}\right)^{2}\right]}{R a\left(a^{2}+n^{2}-1\right)} \xi_{n}=\xi_{n-1}-\xi_{n+1}, & n \geq 2, \\ \frac{2\left[\pi^{2}\left(a^{2}+1\right) \rho-i a \pi+E \pi^{4}\left(a^{2}+1\right)^{2}\right]}{R a^{3}} \xi_{1}+\xi_{2}=0, & n=1 .\end{cases}
$$

Multiplying the $n$th equation of (2.3) and (2.4) by $\bar{\xi}_{n}$ respectively, we obtain

$(2.5) \sum_{n \geq 1-k} \frac{2 \pi^{2}\left[a^{2}+(n+k / 2)^{2}\right]\left\{\operatorname{Re} \rho+E \pi^{2}\left[a^{2}+(n+k / 2)^{2}\right]\right\}}{R a\left[a^{2}+(n+k / 2)^{2}-1\right]}\left|\xi_{n}\right|^{2}=0, k=0,1$.

This implies $\xi_{n} \equiv 0$ whenever Re $\rho \geq 0$ and $a \geq \sqrt{3} / 2$, and Assertion (i) of Theorem 1.1 follows. 
If $k=0,(2.5)$ implies $\xi_{n} \equiv 0$ for all $a>0$ and for all $\rho$ with $\operatorname{Re} \rho \geq 0$. It follows that no bifurcation can arise in the problem governed by (1.1) and (1.2) from an antisymmetric instability.

To address the bifurcation problem, we thus consider the case $a<\sqrt{3} / 2$ and $k=1$. For simplicity, let

$$
d_{n}=\frac{2 \pi^{2}\left(a^{2}+(n+1 / 2)^{2}\right) \rho-2 a \pi i+2 E \pi^{4}\left(a^{2}+(n+1 / 2)^{2}\right)^{2}}{R a\left(a^{2}+(n+1 / 2)^{2}-1\right)} ;
$$

it follows, in particular, that $\operatorname{Re} d_{n}>0$ for $\operatorname{Re} \rho>-E \pi^{2}\left(a^{2}+1 / 4\right)$.

Thus (2.3) and (2.5) become

$$
\begin{cases}d_{0} \xi_{0}+i \xi_{0}+\xi_{1}=0, & n=0, \\ d_{n} \xi_{n}-\xi_{n-1}+\xi_{n+1}=0, & n \geq 1,\end{cases}
$$

and

$$
\sum_{n \geq 0} \operatorname{Re} d_{n}\left|\xi_{n}\right|^{2}=0
$$

It is readily seen that (2.7) implies that $\xi_{n}=0$ for all $n \geq 0$, whenever there exists an $n_{0} \geq 0$ for which $\xi_{n_{0}}=0$.

Thus we may assume that $\xi_{n} \neq 0$ for $n \geq 0$. Hence, we obtain from (2.7) that

$$
\frac{\xi_{1}}{\xi_{0}}=-d_{0}-i, \frac{\xi_{n}}{\xi_{n-1}}=\frac{1}{d_{n}+\frac{\xi_{n+1}}{\xi_{n}}}, \quad n \geq 1 .
$$

It follows therewith from (2.1) that $\rho=\rho(R)$ solves the following continued fraction equation

$$
-d_{0}-i=\frac{1}{d_{1}+\frac{1}{d_{2}+\frac{1}{\ddots}}} .
$$

The above argument implies the following fundamental lemma.

LEMma 2.1. The spectral problem described by (1.5) and (1.2) with unknown eigenfunction $\phi=\phi(x, y)$ given by the expansion (2.1) is equivalent to the difference equation (2.7) with $\left(d_{n}, \xi_{n}\right)$ that satisfy the constraints (2.2), (2.6), (2.8) and (2.9). Moreover, for a nontrivial eigenfunction $\phi$, the corresponding sequence $\left\{\xi_{n}\right\}$ may be represented in the product form

$$
\begin{aligned}
& \xi_{n}=c \gamma_{1} \cdots \gamma_{n}, \quad n \geq 1, \\
& \xi_{0}=c,
\end{aligned}
$$

where $c$ is an arbitrary complex constant and the factors $\gamma_{n}$ are given by

$$
\gamma_{n}=\frac{1}{d_{n}+\frac{1}{d_{n+1}+\frac{1}{\ddots}}} .
$$


3. Existence of A Critical Reynolds Number. In this section, we show the existence of a critical Reynolds number $R_{0}$ and the existence of an eigenvalue $\rho(R)$ such that $\operatorname{Re} \rho\left(R_{0}\right)=0$ and $\operatorname{Im} \rho\left(R_{0}\right)>0$. We begin with the existence of the eigenvalue $\rho$, which may reach, and eventually cross, the imaginary axis of the complex plane.

LEMma 3.1. The spectral problem (1.5) admits a unique pair of complex conjugate eigenvalues $\rho=\rho(R)$ and $\bar{\rho}=\bar{\rho}(R)$ for any $R>0$ and $1 / 2 \leq a \leq 3 / 4$, such that $\operatorname{Re} \rho>-E \pi^{2}\left(a^{2}+1 / 4\right)$.

Proof. ¿From the previous derivation, we see readily that it suffices to show the existence and uniqueness of a function $\rho=\rho(R)$ that satisfies (2.6) and (2.9). Combining these two equations, we may write $\rho(R)$ as

$$
\rho=-E \pi^{2}\left(a^{2}+1 / 4\right)+\frac{i 2 a \pi+i R a\left(3 / 4-a^{2}\right)}{2 \pi^{2}\left(a^{2}+1 / 4\right)}+\frac{\frac{R a\left(3 / 4-a^{2}\right)}{2 \pi^{2}\left(a^{2}+1 / 4\right)}}{d_{1}+\frac{1}{d_{2}+\frac{1}{\ddots}}} .
$$

To derive the existence of the unique pair of eigenvalues that satisfies the required strong inequality, we denote by $\Phi_{R}(\rho)$ the right-hand side of (3.1). It suffices then to show the existence of a fixed point of $\Phi_{R}$ in the complex plane $\mathbb{C}$. Indeed, since $\operatorname{Re} \rho \geq-E \pi^{2}\left(a^{2}+1 / 4\right), R>0$ and $a^{2}<3 / 4$, we have

$$
\operatorname{Re} \Phi_{R}(\rho)>-E \pi^{2}\left(a^{2}+1 / 4\right)
$$

and

$$
\begin{aligned}
\left|\Phi_{R}(\rho)\right| \leq & E \pi^{2}\left(a^{2}+1 / 4\right)+\frac{2 a \pi+R a\left(3 / 4-a^{2}\right)}{2 \pi^{2}\left(a^{2}+1 / 4\right)}+\frac{R a\left(3 / 4-a^{2}\right)}{2 \pi^{2}\left(a^{2}+1 / 4\right)} \frac{1}{\operatorname{Re} d_{1}} \\
\leq & E \pi^{2}\left(a^{2}+1 / 4\right)+\frac{2 a \pi+R a\left(3 / 4-a^{2}\right)}{2 \pi^{2}\left(a^{2}+1 / 4\right)} \\
& +\frac{R^{2} a^{2}\left(3 / 4-a^{2}\right)\left(a^{2}+5 / 4\right)}{4 \pi^{4}\left(a^{2}+1 / 4\right)\left(a^{2}+9 / 4\right)\left[\operatorname{Re} \rho+E\left(a^{2}+9 / 4\right) \pi^{2}\right]} \\
\leq & E \pi^{2}\left(a^{2}+1 / 4\right)+\frac{2 a \pi+R a\left(3 / 4-a^{2}\right)}{2 \pi^{2}\left(a^{2}+1 / 4\right)}+\frac{R^{2} a^{2}\left(3 / 4-a^{2}\right)\left(a^{2}+5 / 4\right)}{8 \pi^{4}\left(a^{2}+1 / 4\right)\left(a^{2}+9 / 4\right) E}
\end{aligned}
$$

Denoting this bound by $K_{R}$, we see that $\Phi_{R}$ maps the closed convex set

$$
C_{R}=\left\{z \in \mathbb{C} ;-E \pi^{2}\left(a^{2}+1 / 4\right) \leq \operatorname{Re} z \leq K_{R}, \quad-K_{R} \leq \operatorname{Re} z \leq K_{R}\right\}
$$

into itself. It follows from Brouwer's fixed point theorem that there exists a value $\rho(R)$ in this closed set such that $\Phi_{R}(\rho(R))=\rho(R)$. This gives the existence of the desired pair of eigenvalues.

In order to show the uniqueness of this pair of eigenvalues, we suppose that there exist two eigenvalues $\rho_{j}=\rho_{j}(R)$ with $j=1,2$ for $R>0$, which are not a complex conjugate pair. Note that, by Lemma 2.1 ,

$$
\gamma_{n}\left(\rho_{j}\right)=\frac{1}{d_{n}+\gamma_{n+1}\left(\rho_{j}\right)}=\lim _{m \rightarrow \infty} \frac{1}{d_{n}+\frac{1}{d_{n+1}+\frac{1}{\ddots \cdot \frac{1}{d_{n+m}}}}},
$$


for $d_{n}=d_{n}\left(\rho_{j}\right)$ defined by (2.6) and $\operatorname{Re} \rho_{j}>-E \pi^{2}\left(a^{2}+1 / 4\right)$ with $j=1,2$. We have

$$
\gamma_{n}\left(\rho_{1}\right)-\gamma_{n}\left(\rho_{2}\right)=-\gamma_{n}\left(\rho_{1}\right) \gamma_{n}\left(\rho_{2}\right)\left[d_{n}\left(\rho_{1}\right)-d_{n}\left(\rho_{2}\right)+\gamma_{n+1}\left(\rho_{1}\right)-\gamma_{n+1}\left(\rho_{2}\right)\right],
$$

and so, by induction,

$$
\begin{aligned}
\gamma_{1}\left(\rho_{1}\right)-\gamma_{1}\left(\rho_{2}\right) & =\sum_{n \geq 1}(-1)^{n}\left[d_{n}\left(\rho_{1}\right)-d_{n}\left(\rho_{2}\right)\right] \eta_{n}\left(\rho_{1}\right) \eta_{n}\left(\rho_{2}\right) \\
& =\sum_{n \geq 1}(-1)^{n} \frac{2 \pi\left[a^{2}+(n+1 / 2)^{2}\right]}{R a\left[\left(a^{2}+(n+1 / 2)^{2}-1\right]\right.} \eta_{n}\left(\rho_{1}\right) \eta_{n}\left(\rho_{2}\right)\left(\rho_{1}-\rho_{2}\right)
\end{aligned}
$$

here $\left\{\eta_{n}\left(\rho_{j}\right)\right\}$ is now the solution specified by Lemma 2.1 such that $\eta_{n}=\xi_{n}$ and $\eta_{0}=c=1$. This yields, for $\rho_{1} \neq \rho_{2}$,

$$
\begin{aligned}
& \left|\rho_{1}-\rho_{2}\right| \\
& =\frac{R a\left(3 / 4-a^{2}\right)}{2 \pi^{2}\left(a^{2}+1 / 4\right)}\left|\gamma_{1}\left(\rho_{1}\right)-\gamma_{1}\left(\rho_{2}\right)\right| \\
& \leq \frac{3 / 4-a^{2}}{2\left(a^{2}+1 / 4\right)} \sum_{n \geq 1} \frac{a^{2}+(n+1 / 2)^{2}}{\left[a^{2}+(n+1 / 2)^{2}-1\right]}\left(\left|\eta_{n}\left(\rho_{1}\right)\right|^{2}+\left|\eta_{n}\left(\rho_{2}\right)\right|^{2}\right)\left|\rho_{1}-\rho_{2}\right| \\
& <\frac{3 / 4-a^{2}}{2 a^{2}+1 / 2} \sum_{i=1}^{2} \sum_{n \geq 1} \frac{\left[a^{2}+(n+1 / 2)^{2}\right]\left[\operatorname{Re} \rho_{i}+E \pi^{2}\left(a^{2}+(n+1 / 2)^{2}\right]\right.}{\left[\operatorname{Re} \rho_{i}+E \pi^{2}\left(a^{2}+1 / 4\right)\right]\left[a^{2}+(n+1 / 2)^{2}-1\right]}\left|\eta_{n}\left(\rho_{i}\right)\right|^{2}\left|\rho_{1}-\rho_{2}\right| \\
& =\left|\rho_{1}-\rho_{2}\right|,
\end{aligned}
$$

where we have used (2.8). This implies $\rho_{1}=\rho_{2}$ and completes the proof of Lemma 3.1. $\square$

Lemma 3.2. Let $\rho=\rho(R)$ with $R>0$ be one of the two complex conjugate eigenvalues deduced in Lemma 3.1. Then we have $\operatorname{Im} \rho(R) \neq 0$.

Proof. Assuming otherwise, i.e. $\operatorname{Im} \rho=0$, we derive a contradiction. To this end, notice that

$$
\begin{aligned}
\left|\frac{\operatorname{Im} d_{n}}{\operatorname{Re} d_{n}}\right| & =\frac{\left|\left(a^{2}+(n+1 / 2)^{2}\right) \operatorname{Im} \rho \pi^{2}-a \pi\right|}{\pi^{2}\left(a^{2}+(n+1 / 2)^{2}\right)\left\{E\left[a^{2}+(n+1 / 2)^{2}\right] \pi^{2}+\operatorname{Re} \rho\right\}} \\
& >\frac{\left|\left(a^{2}+(n+1+1 / 2)^{2}\right) \pi^{2} \operatorname{Im} \rho-a \pi\right|}{\pi^{2}\left(a^{2}+(n+1+1 / 2)^{2}\right)\left\{E\left[a^{2}+(n+1+1 / 2)^{2}\right] \pi^{2}+\operatorname{Re} \rho\right\}} \\
& =\left|\frac{\operatorname{Im} d_{(n+1)}}{\operatorname{Re} d_{(n+1)}}\right| .
\end{aligned}
$$

This, together with induction on $n$, implies that

$$
\frac{\left|\operatorname{Im} \gamma_{1}\right|}{\operatorname{Re} \gamma_{1}}<\left|\frac{\operatorname{Im} d_{1}}{\operatorname{Re} d_{1}}\right|
$$

Thus we have

$$
\left|\frac{\operatorname{Im}\left(-d_{0}-i\right)}{-\operatorname{Re} d_{0}}\right|<\left|\frac{\operatorname{Im} d_{1}}{\operatorname{Re} d_{1}}\right|
$$

and so

$$
\frac{\left|2\left(a^{2}+1 / 4\right) \pi^{2} \operatorname{Im} \rho-2 a \pi-R a\left(3 / 4-a^{2}\right)\right|}{\left(a^{2}+1 / 4\right) \operatorname{Re} \rho+E\left(a^{2}+1 / 4\right)^{2} \pi^{2}}<\frac{2\left|\left(a^{2}+9 / 4\right) \pi^{2} \operatorname{Im} \rho-a \pi\right|}{\left(a^{2}+9 / 4\right) \operatorname{Re} \rho+E\left(a^{2}+9 / 4\right)^{2} \pi^{2}},
$$


and therefore,

$$
\begin{aligned}
\frac{\left|2\left(a^{2}+1 / 4\right) \pi^{2} \operatorname{Im} \rho-2 a \pi-R a\left(3 / 4-a^{2}\right)\right|}{2\left|\left(a^{2}+9 / 4\right) \pi^{2} \operatorname{Im} \rho-a \pi\right|} & <\frac{\left(a^{2}+1 / 4\right)\left[\operatorname{Re} \rho+E\left(a^{2}+1 / 4\right) \pi^{2}\right]}{\left(a^{2}+9 / 4\right)\left[\operatorname{Re} \rho+E\left(a^{2}+9 / 4\right) \pi^{2}\right]} \\
& \leq \frac{a^{2}+1 / 4}{a^{2}+9 / 4}
\end{aligned}
$$

where we have used the condition $\operatorname{Re} \rho+E\left(a^{2}+1 / 4\right) \pi^{2}>0$. This implies

$$
1+\frac{R a\left(3 / 4-a^{2}\right)}{2\left|\left(a^{2}+9 / 4\right) \pi^{2} \operatorname{Im} \rho-a \pi\right|}<\frac{a^{2}+1 / 4}{a^{2}+9 / 4}+\frac{2 \pi^{2}|\operatorname{Im} \rho|}{\left|\left(a^{2}+9 / 4\right) \pi^{2} \operatorname{Im} \rho-a \pi\right|}<1 .
$$

This leads to a contradiction and hence $\operatorname{Im} \rho>0$. The proof of Lemma 3.2 is complete.

$\square$

Lemma 3.3. For one of the two eigenvalues obtained in Lemma 3.1, we have

$$
\frac{1}{26}<\frac{2 \pi^{2}}{a} \limsup _{R \rightarrow \infty} \frac{\operatorname{Im} \rho(R)}{R}<\frac{3}{2} .
$$

Proof. By (2.8), (2.9) and Lemma 2.1, we have

$$
-\operatorname{Re} d_{0}\left|\xi_{0}\right|^{2}>\operatorname{Re} d_{1}\left|\xi_{1}\right|^{2}
$$

or

$$
-\operatorname{Re} d_{0}>\operatorname{Re} d_{1}\left|d_{0}+i\right|^{2}
$$

Together with (2.6), this implies

$$
\frac{\left(a^{2}+1 / 4\right)^{2}}{3 / 4-a^{2}}>\frac{\left(a^{2}+9 / 4\right)^{2}}{a^{2}+5 / 4}\left[\frac{2 \pi^{2}\left(a^{2}+1 / 4\right) \operatorname{Im} \rho-2 a \pi}{R a\left(3 / 4-a^{2}\right)}-1\right]^{2},
$$

and so

$$
\frac{\left(a^{2}+1 / 4\right)^{2}\left(a^{2}+5 / 4\right)}{\left(3 / 4-a^{2}\right)\left(a^{2}+9 / 4\right)^{2}} \geq\left[\frac{2 \pi^{2}\left(a^{2}+1 / 4\right)}{a\left(3 / 4-a^{2}\right)} \limsup _{R \rightarrow \infty} \frac{\operatorname{Im} \rho}{R}-1\right]^{2} .
$$

Therefore,

$$
\frac{1}{26} \leq \frac{2 \pi^{2}}{a} \limsup _{R \rightarrow \infty} \frac{\operatorname{Im} \rho}{R} \leq 3 / 2
$$

for $1 / 2 \leq a \leq 3 / 4$. The proof of Lemma 3.3 is thus complete.

Now we prove the main result of this section.

THEOREM 3.4. Let $\rho(R)$ be either one of the two eigenvalues obtained in Lemma 3.1. Then there exists a critical Reynolds number $R_{0}$ such that $\operatorname{Re} \rho\left(R_{0}\right)=0$ and $\operatorname{Im} \rho\left(R_{0}\right) \neq 0$.

Proof. First, we prove the smoothness of $\rho(R)$ by using the implicit function theorem. Define the function

$$
F(\rho, R)=i+d_{0}+\frac{1}{d_{1}+\frac{1}{d_{2}+\frac{1}{\ddots}}}
$$


with $d_{n}=d_{n}(\rho, R)$ given by $(2.6)$. We see immediately that $F(\rho(R), R)=0$, due to (2.9) and Lemma 3.1. It follows from (3.4) that

$$
\frac{\partial F(\rho, R)}{\partial \rho}=\sum_{n \geq 0}(-1)^{n} \frac{\partial d_{n}}{\partial \rho} \eta_{n}^{2}=\sum_{n \neq 0}(-1)^{n} \frac{2 \pi\left[a^{2}+(n+1 / 2)^{2}\right]}{R a\left[\left(a^{2}+(n+1 / 2)^{2}-1\right]\right.} \eta_{n}^{2},
$$

where $\left\{\eta_{n}\right\}$ is now the solution specified by Lemma 2.1 such that $\eta_{0}=1$. Hence we have, by (2.8), that

$$
\begin{aligned}
\left|\frac{\partial F(\rho, R)}{\partial \rho}\right| \geq & \frac{2 \pi^{2}\left(a^{2}+1 / 4\right)}{R a\left(3 / 4-a^{2}\right)}\left|\eta_{0}\right|^{2}-\sum_{n \geq 1} \frac{2 \pi^{2}\left[a^{2}+(n+1 / 2)^{2}\right]}{R a\left[a^{2}+(n+1 / 2)^{2}-1\right]}\left|\eta_{n}\right|^{2} \\
= & \frac{2 \pi^{2}}{R a} \sum_{n \geq 1} \frac{\left[a^{2}+(n+1 / 2)^{2}\right]\left[\operatorname{Re} \rho+E \pi^{2}\left(a^{2}+(n+1 / 2)^{2}\right]\right.}{\left[\operatorname{Re} \rho+E \pi^{2}\left(a^{2}+1 / 4\right)\right]\left[a^{2}+(n+1 / 2)^{2}-1\right]}\left|\eta_{n}\right|^{2} \\
& -\sum_{n \geq 1} \frac{2 \pi^{2}\left[a^{2}+(n+1 / 2)^{2}\right]}{R a\left[a^{2}+(n+1 / 2)^{2}-1\right]}\left|\eta_{n}\right|^{2} \\
= & \frac{2 \pi^{4} E}{\operatorname{Re} \rho+E \pi^{2}\left(a^{2}+1 / 4\right)} \sum_{n \geq 1} \frac{\left[a^{2}+(n+1 / 2)^{2}\right](n+1) n}{R a\left[a^{2}+(n+1 / 2)^{2}-1\right]}\left|\eta_{n}\right|^{2}>0
\end{aligned}
$$

Thus, by the implicit function theorem, $\rho=\rho(R)$ is smooth.

Next, letting $R \rightarrow 0$ in (2.6) and (2.9), we see that

$$
\lim _{R \rightarrow 0} \operatorname{Re} \rho(R)=-E \pi^{2}\left(a^{2}+1 / 4\right) .
$$

Finally, by Lemma 3.2 and the smoothness of $\rho(R)$, it suffices to show that

$$
\limsup _{R \rightarrow \infty} \operatorname{Re} \rho(R)>0 .
$$

To do so, we suppose that $\lim \sup _{R \rightarrow \infty} \operatorname{Re} \rho(R) \leq 0$, which will lead to a contradiction.

Without loss of generality, by Lemma 3.3, we may suppose that

$$
\lim _{R \rightarrow \infty} \operatorname{Re} \rho(R)=\mu, \lim _{R \rightarrow \infty} \frac{2 \pi^{2} \operatorname{Im} \rho(R)}{R a}=\nu
$$

for some constants $\mu$ and $\nu$. Otherwise, we may consider instead a subsequence $\left\{R_{n}\right\}$ that converges to these values.

Applying (2.9) and Lemma 2.1 yields

$$
-d_{0}-i=\frac{1}{d_{1}+\frac{1}{d_{2}+\gamma_{3}}} .
$$

Hence

$$
\begin{aligned}
\lim _{R \rightarrow \infty} \gamma_{3} & =\frac{1}{-\frac{1}{\lim _{R \rightarrow \infty} i \operatorname{Im} d_{0}+i}-\lim _{R \rightarrow \infty} i \operatorname{Im} d_{1}}-\lim _{R \rightarrow \infty} i \operatorname{Im} d_{2} \\
& =i \frac{\lim _{R \rightarrow \infty} \operatorname{Im} d_{0}+1}{\lim _{R \rightarrow \infty} \operatorname{Im} d_{1}\left(\lim _{R \rightarrow \infty} \operatorname{Im} d_{0}+1\right)-1}-\lim _{R \rightarrow \infty} i \operatorname{Im} d_{2}
\end{aligned}
$$


or

$$
\lim _{R \rightarrow \infty}\left(\operatorname{Im} \gamma_{3}+\operatorname{Im} d_{2}\right)=\frac{\lim _{R \rightarrow \infty} \operatorname{Im} d_{0}+1}{\lim _{R \rightarrow \infty} \operatorname{Im} d_{1}\left(\operatorname{Im} d_{0}+1\right)-1} .
$$

Furthermore, it follows from (3.9) that

$$
-d_{0}-i=\frac{d_{2}+\gamma_{3}}{d_{1}\left(d_{2}+\gamma_{3}\right)+1}=\frac{\left(d_{2}+\gamma_{3}\right)\left(\overline{d_{1}\left(d_{2}+\gamma_{3}\right)}+1\right)}{\left|d_{1}\left(d_{2}+\gamma_{3}\right)+1\right|^{2}},
$$

and thus

$$
\begin{aligned}
& -\operatorname{Re} d_{0}\left|d_{1}\left(d_{2}+\gamma_{3}\right)+1\right|^{2} \\
& =\operatorname{Re}\left(d_{2}+\gamma_{3}\right) \operatorname{Re}\left(d_{1}\left(d_{2}+\gamma_{3}\right)+1\right)-\operatorname{Im}\left(d_{2}+\gamma_{3}\right) \operatorname{Im} \overline{d_{1}\left(d_{2}+\gamma_{3}\right)} \\
& =\operatorname{Re}\left(d_{2}+\gamma_{3}\right)\left(\operatorname{Re} d_{1} \operatorname{Re}\left(d_{2}+\gamma_{3}\right)+1\right)+\operatorname{Re} d_{1}\left(\operatorname{Im} d_{2}+\operatorname{Im} \gamma_{3}\right)^{2} .
\end{aligned}
$$

Multiplying the last equation by $R$ and passing to the limit $R \rightarrow \infty$, we obtain, after using (3.8), (3.10), (3.11) and the positivity of $\operatorname{Re} \gamma_{3}$,

$$
\begin{aligned}
\lim _{R \rightarrow \infty} R\left|\operatorname{Re} d_{0}\right|= & \frac{\lim _{R \rightarrow \infty} R \operatorname{Re}\left(d_{2}+\gamma_{3}\right)+\lim _{R \rightarrow \infty} R \operatorname{Re} d_{1} \lim _{R \rightarrow \infty}\left(\operatorname{Im} d_{2}+\operatorname{Im} \gamma_{3}\right)^{2}}{\left|\lim _{R \rightarrow \infty} \operatorname{Im} d_{1} \lim _{R \rightarrow \infty}\left(\operatorname{Im} d_{2}+\operatorname{Im} \gamma_{3}\right)-1\right|^{2}} \\
\geq & \frac{\lim _{R \rightarrow \infty} R \operatorname{Re} d_{2}+\lim _{R \rightarrow \infty} R \operatorname{Re} d_{1} \lim _{R \rightarrow \infty}\left(\operatorname{Im} d_{2}+\operatorname{Im} \gamma_{3}\right)^{2}}{\left|\lim _{R \rightarrow \infty} \operatorname{Im} d_{1} \lim _{R \rightarrow \infty}\left(\operatorname{Im} d_{2}+\operatorname{Im} \gamma_{3}\right)-1\right|^{2}} \\
= & \lim _{R \rightarrow \infty} R \operatorname{Re} d_{1}\left(\lim _{R \rightarrow \infty} \operatorname{Im} d_{0}+1\right)^{2} \\
& +\lim _{R \rightarrow \infty} R \operatorname{Re} d_{2}\left(1-\lim _{R \rightarrow \infty} \operatorname{Im} d_{1}\left(\operatorname{Im} d_{0}+1\right)\right)^{2} .
\end{aligned}
$$

That is,

$$
\begin{aligned}
& \frac{a^{2}+1 / 4}{3 / 4-a^{2}}\left[\mu+E\left(a^{2}+1 / 4\right) \pi^{2}\right] \\
& \geq \frac{a^{2}+9 / 4}{a^{2}+5 / 4}\left[\mu+E\left(a^{2}+9 / 4\right) \pi^{2}\right]\left(1-\frac{a^{2}+1 / 4}{3 / 4-a^{2}} \nu\right)^{2} \\
& \quad+\frac{a^{2}+25 / 4}{a^{2}+21 / 4}\left[\mu+E\left(a^{2}+25 / 4\right) \pi^{2}\right]\left[\frac{a^{2}+9 / 4}{a^{2}+5 / 4}\left(1-\frac{a^{2}+1 / 4}{3 / 4-a^{2}} \nu\right) \nu-1\right]^{2} .
\end{aligned}
$$

This together with the condition $-E\left(a^{2}+1 / 4\right) \pi^{2} \leq \mu \leq 0$ implies

$$
\begin{aligned}
\frac{a^{2}+1 / 4}{3 / 4-a^{2}} \geq & \frac{a^{2}+9 / 4}{a^{2}+5 / 4}\left(1-\frac{a^{2}+1 / 4}{3 / 4-a^{2}} \nu\right)^{2} \\
& +\frac{a^{2}+25 / 4}{a^{2}+21 / 4}\left[1-\frac{a^{2}+9 / 4}{a^{2}+5 / 4}\left(1-\frac{a^{2}+1 / 4}{3 / 4-a^{2}} \nu\right) \nu\right]^{2},
\end{aligned}
$$

and so, by an elementary calculation,

$$
\frac{\left(a^{2}+1 / 4\right)^{2}}{3 / 4-a^{2}} \geq \frac{\left(a^{2}+9 / 4\right)^{2}}{a^{2}+5 / 4}\left(1-\frac{a^{2}+1 / 4}{3 / 4-a^{2}} \nu\right)^{2}
$$




$$
\begin{aligned}
& +\frac{\left(a^{2}+25 / 4\right)^{2}}{a^{2}+21 / 4}\left[1-\frac{a^{2}+9 / 4}{a^{2}+5 / 4}\left(1-\frac{a^{2}+1 / 4}{3 / 4-a^{2}} \nu\right) \nu\right]^{2} \\
> & \frac{\left(a^{2}+25 / 4\right)^{2}}{a^{2}+21 / 4}\left[1-\frac{\left(a^{2}+9 / 4\right)\left(3 / 4-a^{2}\right)}{4\left(a^{2}+5 / 4\right)\left(a^{2}+1 / 4\right)}\right]^{2} \\
> & \frac{\left(a^{2}+1 / 4\right)^{2}}{3 / 4-a^{2}}
\end{aligned}
$$

for $1 / 2 \leq a \leq 3 / 4$. This leads to a contradiction. Hence the $\lim _{R \rightarrow \infty} \operatorname{Re} \rho>0$. The proof of Theorem 3.1 is thus complete.

4. Spectral Simplicity Condition. This section is devoted to the simplicity of the two eigenvalues that cross the imaginary axis. We prove the following theorem:

THEOREM 4.1. Let the critical Reynolds number $R_{0}$ and the eigenvalue $\rho=\rho\left(R_{0}\right)$ with Im $\rho>0$ be as shown to exist in Theorem 3.4. Then this eigenvalue is simple, i.e.,

$$
\operatorname{dim} \bigcup_{n \geq 1}\left\{\phi \in H^{4} ; L^{n} \phi=0\right\}=1
$$

where $L=L(\rho)$ is the linear operator defined in (1.5).

Proof. We introduce the invariant subspaces of the spectral problem (1.5) for any integer $m$

$$
E_{m, k}=\left\{\phi \in H^{4} ; \psi(x, y)=\sum_{n \geq 1-k} i^{n} \phi_{n} e^{i m a \pi x} \sin (n+k / 2) \pi y\right\}, k=0,1 .
$$

Hence the simplicity condition holds true provided that the following assertions are valid:

$$
\operatorname{dim} \bigcup_{n \geq 1}\left\{\phi \in E_{m, k} ; L^{n} \phi=0\right\}=0
$$

for $(m, k) \neq(1,1)$, and

$$
\operatorname{dim} \bigcup_{n \geq 1}\left\{\phi \in E_{1,1} ; L^{n} \phi=0\right\}=1
$$

Let us begin with the proof of (4.2). By the derivation of (2.5), we see that, for an unknown eigenfunction

$$
\phi=e^{i m a \pi x} \sum_{n \geq 1-k} \frac{i^{n}}{m^{2} a^{2}+(n+k / 2)^{2}-1} \phi_{n} \sin (n+k / 2) \pi y
$$

of the spectral problem, (4.2) implies the validity of (2.5), with a replaced by $a m$. That is,

$$
\sum_{n \geq 1-k} \frac{\left[m^{2} a^{2}+(n+k / 2)^{2}\right]\left\{\operatorname{Re} \rho+E \pi^{2}\left[m^{2} a^{2}+(n+k / 2)^{2}\right]\right\}}{m^{2} a^{2}+(n+k / 2)^{2}-1}\left|\phi_{n}\right|^{2}=0
$$

for $k=0,1$. If $(m, k) \neq( \pm 1,1)$, it is easy to see that the expansion coefficients $\phi_{n} \equiv 0$ and hence $\phi(x, y) \equiv 0$. This gives $(4.2)$ with $(m, k) \neq( \pm 1,1)$. 
If $(m, k)=(-1,1)$, it follows from Lemma 2.1 and Theorem 3.4 that

$$
\operatorname{dim}\left\{\phi \in E_{-1,1} ; L\left(\rho^{\prime}\right) \phi=0\right\}=1
$$

if and only if $\rho^{\prime}=\bar{\rho}$; this implies $\operatorname{Im} \rho^{\prime}<0$, due to the assumption of Theorem 4.1 and Lemma 3.2. Hence we obtain $(4.2)$ for $(m, k)=(-1,1)$.

In order to prove (4.3), we first note from Lemma 2.1 and Theorem 3.4 that

$$
\operatorname{dim}\left\{\phi \in E_{1,1} ; L \phi=0\right\}=1 .
$$

By induction, it remains to show

$$
\operatorname{dim}\left\{\phi \in E_{1,1} ; L^{2} \phi=0\right\}=1 .
$$

Indeed, rewrite the equation $L^{2} \phi=0$ as

$$
L \chi=0,
$$

after setting

$$
L \phi=\chi
$$

for some $\phi \in E_{1,1}$. It remains to show that $\phi \equiv 0$. Following the derivation of (2.7), we obtain the equivalent formulation of (4.5) and (4.6)

$$
\begin{cases}d_{n} \xi_{n}^{\prime}-\xi_{n-1}^{\prime}+\xi_{n+1}^{\prime}=\xi_{n}, & n \geq 1 \\ d_{n} \xi_{n}-\xi_{n-1}+\xi_{n+1}=0, & n \geq 1 \\ d_{0} \xi_{0}^{\prime}+i \xi_{0}^{\prime}+\xi_{1}^{\prime}=\xi_{0}, & n=0 \\ d_{0} \xi_{0}+i \xi_{0}+\xi_{1}=0, & n=0\end{cases}
$$

here $d_{n}$ is defined by (2.6) and $\left\{\xi_{n} / d_{n}\right\},\left\{\xi_{n}^{\prime}\right\} \in l^{2}$, while $l^{2}$ denotes the Hilbert space

$$
l^{2}=\left\{\left\{\xi_{n}\right\} ;\left\|\left\{\xi_{n}\right\}\right\|_{l^{2}}^{2}=\sum_{n \geq 0} n^{2}\left|\xi_{n}\right|^{2}<\infty\right\} .
$$

Define an operator $M: l^{2} \mapsto l^{2}$ such that $M\left\{\xi_{n}\right\}=\left\{\eta_{n}\right\}$ with

$$
\eta_{n}=\frac{1}{d_{n}}\left(\xi_{n+1}-\xi_{n-1}\right), \quad \eta_{0}=\frac{1}{d_{0}}\left(i \xi_{0}+\xi_{1}\right), \quad n \geq 1
$$

Equation (4.7) becomes

$$
\left\{\begin{aligned}
(1+M)\left\{\xi_{n}^{\prime}\right\} & =\left\{\frac{\xi_{n}}{d_{n}}\right\}, \\
(1+M)\left\{\xi_{n}\right\} & =0 .
\end{aligned}\right.
$$

We see that $M$ is compact in $l^{2}$. It follows from Riesz-Schauder theory (also called the Fredholm alternative principle; see Theorem 5.3 in [10]) that (4.8) is solvable if and only if

$$
\sum_{n \geq 0} \frac{\xi_{n} \bar{\zeta}_{n}}{d_{n}}=0
$$


whenever $\left\{\zeta_{n}\right\} \in l^{2}$ is a nontrivial solution of the dual equation

$$
(1+\bar{M})\left\{\zeta_{n}\right\}=0 .
$$

Here $\bar{M}$ is the dual operator of $M$.

Such a nontrivial solution of (4.10) is given by

$$
\begin{aligned}
& \zeta_{n}+\frac{\zeta_{n-1}}{\bar{d}_{n-1}}-\frac{\zeta_{n+1}}{\bar{d}_{n+1}}=0, n \geq 1, \\
& \zeta_{0}-\frac{i \zeta_{0}}{\bar{d}_{0}}-\frac{\zeta_{1}}{\bar{d}_{1}}=0, n=0 .
\end{aligned}
$$

This becomes, by setting $\hat{\zeta}_{n}=(-1)^{n} \bar{\zeta}_{n} / d_{n}$,

$$
\begin{aligned}
& d_{n} \hat{\zeta}_{n}-\hat{\zeta}_{n-1}+\hat{\zeta}_{n+1}=0, n \geq 1, \\
& d_{0} \hat{\zeta}_{0}+i \hat{\zeta}_{0}+\hat{\zeta}_{1}=0, n=0 .
\end{aligned}
$$

By Lemma 2.1 and Theorem 3.4, we have

$$
\hat{\zeta}_{n}=(-1)^{n} \frac{\bar{\zeta}_{n}}{d_{n}}=c \xi_{n}, \quad n \geq 0
$$

for some constant $c \neq 0$. Thus (4.9) becomes

$$
\sum_{n \geq 0} \frac{\xi_{n} \bar{\zeta}_{n}}{d_{n}}=c \sum_{n \geq 0}(-1)^{n} \xi_{n}^{2}=0 .
$$

Hence, it follows from (2.8) that

$$
\begin{aligned}
0 & =\left|\sum_{n \geq 0}(-1)^{n} \xi_{n}^{2}\right| \\
& \geq\left|\xi_{0}\right|^{2}-\sum_{n \geq 1}\left|\xi_{n}\right|^{2} \\
& =\frac{1}{\left|\operatorname{Re} d_{0}\right|} \sum_{n \geq 1}\left(\operatorname{Re} d_{n}-\left|\operatorname{Re} d_{0}\right|\right)\left|\xi_{n}\right|^{2} .
\end{aligned}
$$

Since $\operatorname{Re} d_{n}-\left|\operatorname{Re} d_{0}\right|>0$ for $1 / 2 \leq a \leq 3 / 4$, we thus have $\left\{\xi_{n}\right\}=0$ and hence (4.4). The proof of Theorem 4.1 is complete.

5. Transversal Crossing Condition. The objective of this section is to show that the pair of the eigenvalues $\{\rho(R), \bar{\rho}(R)\})$ crosses the imaginary axis transversally and away from the origin. This result reads as follows.

TheOrem 5.1. Let $\rho=\rho\left(R_{0}\right)$ and $R_{0}$ be as characterized in Lemma 2.1 and Theorems 3.4 and 4.1. Then the transversal crossing condition

$$
R e \frac{d \rho\left(R_{0}\right)}{d R}>0
$$

holds true, provided that $E>c_{0}$ for some constant $c_{0}>0$.

Proof. Recalling the definition of the function $F=F(\rho, R)$ in (3.5), we see that the eigenvalue $\rho=\rho(R)$ satisfies the equation $F(\rho(R), R)=0$, and so

$$
\frac{\partial F}{\partial \rho} \frac{\mathrm{d} \rho(R)}{d R}+\frac{\partial F}{\partial R}=0
$$


It follows from the derivation of (3.6) that

$$
\frac{\partial F}{\partial R}=-\frac{1}{R} \sum_{n \geq 0}(-1)^{n} d_{n} \eta_{n}^{2} .
$$

Thus, by (2.6) with $R=R_{0},(3.6)$, and (3.7), we have

$$
\begin{aligned}
\pi R_{0} \operatorname{Re} \frac{\mathrm{d} \rho\left(R_{0}\right)}{\mathrm{d} R} & =\pi \operatorname{Re} \frac{\sum_{n \geq 0}(-1)^{n} d_{n} \eta_{n}^{2}}{\sum_{n \geq 0}(-1)^{n} \frac{\partial d_{n}}{\partial \rho} \eta_{n}^{2}} \\
& =\operatorname{Re} \frac{\sum_{n \geq 0}(-1)^{n} \frac{\pi^{3} E\left[a^{2}+(n+1 / 2)^{2}\right]^{2}-i a}{a^{2}+(n+1 / 2)^{2}-1} \eta_{n}^{2}}{\sum_{n \geq 0}(-1)^{n} \frac{a^{2}+(n+1 / 2)^{2}}{a^{2}+(n+1 / 2)^{2}-1} \eta_{n}^{2}} .
\end{aligned}
$$

This equals, after setting $a^{2}+(n+1 / 2)^{2}=\beta_{n}$,

$$
\begin{aligned}
& \operatorname{Re} \frac{\sum_{n \geq 0}(-1)^{n} \frac{\pi^{3} E \beta_{n}^{2}-i a}{\beta_{n}-1} \eta_{n}^{2}}{\sum_{n \geq 0}(-1)^{n} \frac{\beta_{n}}{\beta_{n}-1} \eta_{n}^{2}} \\
& =\pi^{3} E \beta_{0}+\operatorname{Re} \frac{\sum_{n \geq 0}(-1)^{n} \frac{\pi^{3} E \beta_{n}\left(\beta_{n}-\beta_{0}\right)}{\beta_{n}-1} \eta_{n}^{2}+\frac{i a}{\beta_{0}} \sum_{n \geq 1}(-1)^{n} \frac{\beta_{n}-\beta_{0}}{\beta_{n}-1} \eta_{n}^{2}}{\sum_{n \geq 0}(-1)^{n} \frac{\beta_{n}}{\beta_{n}-1} \eta_{n}^{2}} \\
& =\pi^{3} E \beta_{0}+\sum_{n \geq 1}(-1)^{n} \frac{\pi^{3} E \beta_{n}\left(\beta_{n}-\beta_{0}\right)}{\beta_{n}-1} \operatorname{Re}\left(\xi_{n}^{2}\right)-\frac{a}{\beta_{0}} \sum_{n \geq 1}(-1)^{n} \frac{\beta_{n}-\beta_{0}}{\beta_{n}-1} \operatorname{Im}\left(\xi_{n}^{2}\right),
\end{aligned}
$$

where the solution $\left\{\xi_{n}\right\}$ is chosen such that

$$
\xi_{0}^{2}=\frac{1}{\sum_{n \geq 0}(-1)^{n} \frac{\beta_{n}}{\beta_{n}-1} \eta_{n}^{2}} .
$$

We thus have

$$
\begin{aligned}
\pi & R_{0} \operatorname{Re} \frac{\mathrm{d} \rho\left(R_{0}\right)}{\mathrm{d} R} \\
= & \pi^{3} E \beta_{0}\left[1-\frac{1}{\beta_{0}} \sum_{n \geq 1} \frac{\beta_{n}\left(\beta_{n}-\beta_{0}\right)}{\beta_{n}-1}\left|\xi_{n}\right|^{2}\right]-\frac{a}{\beta_{0}} \sum_{n \geq 1}(-1)^{n} \frac{\beta_{n}-\beta_{0}}{\beta_{n}-1} \operatorname{Im}\left(\xi_{n}^{2}\right) \\
& +\pi^{3} E \sum_{n \geq 1} \frac{\beta_{n}\left(\beta_{n}-\beta_{0}\right)}{\beta_{n}-1}\left[\left|\xi_{n}\right|^{2}+(-1)^{n} \operatorname{Re}\left(\xi_{n}^{2}\right)\right] .
\end{aligned}
$$


Furthermore, by (2.8) and (5.1), we have

$$
\begin{aligned}
& 1-\frac{1}{\beta_{0}} \sum_{n \geq 1} \frac{\beta_{n}\left(\beta_{n}-\beta_{0}\right)}{\beta_{n}-1}\left|\xi_{n}\right|^{2} \\
& =1-\frac{1}{\beta_{0}} \sum_{n \geq 1} \frac{\beta_{n}^{2}}{\beta_{n}-1}\left|\xi_{n}\right|^{2}+\sum_{n \geq 1} \frac{\beta_{n}}{\beta_{n}-1}\left|\xi_{n}\right|^{2} \\
& =\left|\xi_{0}\right|^{2}\left[\left|\sum_{n \geq 0}(-1)^{n} \frac{\beta_{n}}{\beta_{n}-1} \eta_{n}^{2}\right|+\sum_{n \geq 1} \frac{\beta_{n}}{\beta_{n}-1}\left|\eta_{n}\right|^{2}+\frac{\beta_{0}}{\beta_{0}-1}\right] \\
& \geq\left|\xi_{0}\right|^{2}\left[\left|\sum_{n \geq 0}(-1)^{n} \frac{\beta_{n}}{\beta_{n}-1} \operatorname{Re}\left(\eta_{n}^{2}\right)\right|+\sum_{n \geq 1} \frac{\beta_{n}}{\beta_{n}-1}\left|\eta_{n}\right|^{2}+\frac{\beta_{0}}{\beta_{0}-1}\right] \\
& \geq\left|\xi_{0}\right|^{2} \sum_{n \geq 1} \frac{\beta_{n}}{\beta_{n}-1}\left|\eta_{n}\right|^{2}-\left|\xi_{0}\right|^{2}\left|\sum_{n \geq 1}(-1)^{n} \frac{\beta_{n}}{\beta_{n}-1} \operatorname{Re}\left(\eta_{n}^{2}\right)\right| \\
& \geq\left|\xi_{0}\right|^{2} \frac{\beta_{1}}{\beta_{1}-1}\left(\left|\eta_{1}\right|^{2}-\left|\operatorname{Re}\left(\eta_{1}^{2}\right)\right|\right),
\end{aligned}
$$

and

$$
\begin{aligned}
\left|\sum_{n \geq 1}(-1)^{n} \frac{\beta_{n}-\beta_{0}}{\beta_{n}-1} \operatorname{Im}\left(\xi_{n}^{2}\right)\right| & \leq \sum_{n \geq 1} \frac{\beta_{n}-\beta_{0}}{\beta_{n}-1}\left|\operatorname{Im}\left(\xi_{n}^{2}\right)\right| \\
& \leq \frac{1}{\beta_{1}} \sum_{n \geq 1} \frac{\beta_{n}^{2}}{\beta_{n}-1}\left|\xi_{n}\right|^{2}=\frac{\beta_{0}^{2}}{\beta_{1}\left(1-\beta_{0}\right)}\left|\xi_{0}\right|^{2} .
\end{aligned}
$$

Collecting terms and using (2.9) and Lemma 2.1, we have

$$
\begin{aligned}
& \frac{R_{0}}{\pi^{2}\left|\xi_{0}\right|^{2}} \operatorname{Re} \frac{\mathrm{d} \rho\left(R_{0}\right)}{\mathrm{d} R} \\
\geq & E \frac{\beta_{1} \beta_{0}}{\beta_{1}-1}\left(\left|\eta_{1}\right|^{2}-\left|\operatorname{Re} \eta_{1}^{2}\right|\right)+E \frac{\beta_{1}\left(\beta_{1}-\beta_{0}\right)}{\beta_{1}-1}\left(\left|\eta_{1}\right|^{2}-\frac{\operatorname{Re} \xi_{1}^{2}}{\left|\xi_{0}\right|^{2}}\right)-\frac{a \beta_{0}}{\pi^{3} \beta_{1}\left(1-\beta_{0}\right)} \\
\geq & E \beta_{0}\left(\left|\eta_{1}\right|^{2}-\left|\operatorname{Re} \eta_{1}^{2}\right|\right)+E\left(\beta_{1}-\beta_{0}\right)\left(\left|\eta_{1}\right|^{2}-\frac{\operatorname{Re} \xi_{1}^{2}}{\left|\xi_{0}\right|^{2}}\right)-\frac{a \beta_{0}}{\pi^{3} \beta_{1}\left(1-\beta_{0}\right)} \\
= & E \beta_{0}\left(\left|d_{0}+i\right|^{2}-\left|\operatorname{Re}\left(d_{0}+i\right)^{2}\right|\right) \\
& +2 E\left(|d+i|^{2}-\frac{\operatorname{Re}\left[\left(d_{0}+i\right)^{2} \xi_{0}^{2}\right]}{\left|\xi_{0}\right|^{2}}\right)-\frac{a \beta_{0}}{\pi^{3} \beta_{1}\left(1-\beta_{0}\right)} .
\end{aligned}
$$

Let us now apply the following lemma, whose proof will be given at the end of this section.

Lemma 5.2. One of the two following estimates

$$
\liminf _{E \rightarrow \infty} \frac{E}{R_{0}}>0,
$$

or

$$
\liminf _{E \rightarrow \infty}\left|\operatorname{Re} d_{0}\right|>0
$$


holds true.

If $\left|1+\operatorname{Im} d_{0}\right|>\left|\operatorname{Re} d_{0}\right|$, we have

$$
\begin{aligned}
\frac{R_{0}}{\pi^{2}\left|\xi_{0}\right|^{2}} \operatorname{Re} \frac{\mathrm{d} \rho\left(R_{0}\right)}{\mathrm{d} R} & \geq E \beta_{0}\left(\left|d_{0}+i\right|^{2}-\left|\operatorname{Re}\left(d_{0}+i\right)^{2}\right|\right)-\frac{a \beta_{0}}{\pi^{3} \beta_{1}\left(1-\beta_{0}\right)} \\
& =E \beta_{0}\left(\left|d_{0}+i\right|^{2}-\left|\left(\operatorname{Re} d_{0}\right)^{2}-\left(1+\operatorname{Im} d_{0}\right)^{2}\right|\right)-\frac{a \beta_{0}}{\pi^{3} \beta_{1}\left(1-\beta_{0}\right)} \\
& =2 E \beta_{0}\left(\operatorname{Re} d_{0}\right)^{2}-\frac{a \beta_{0}}{\pi^{3} \beta_{1}\left(1-\beta_{0}\right)} ;
\end{aligned}
$$

the latter is positive due to Lemma 5.1, after letting $E>c_{0}$ for some positive constant $c_{0}$.

If $\left|\operatorname{Re} d_{0}\right| \geq\left|1+\operatorname{Im} d_{0}\right|>\left|\operatorname{Re} d_{0}\right| / 4$, we see that

$$
\begin{aligned}
\frac{R_{0}}{\pi^{2}\left|\xi_{0}\right|^{2}} \operatorname{Re} \frac{\mathrm{d} \rho\left(R_{0}\right)}{\mathrm{d} R} & \geq E \beta_{0}\left(\left|d_{0}+i\right|^{2}-\left|\operatorname{Re}\left(d_{0}+i\right)^{2}\right|\right)-\frac{a \beta_{0}}{\pi^{3} \beta_{1}\left(1-\beta_{0}\right)} \\
& =2 E \beta_{0}\left(1+\operatorname{Im} d_{0}\right)^{2}-\frac{a \beta_{0}}{\pi^{3} \beta_{1}\left(1-\beta_{0}\right)} \\
& \geq \frac{1}{2} E \beta_{0}\left(\operatorname{Re} d_{0}\right)^{2}-\frac{a \beta_{0}}{\pi^{3} \beta_{1}\left(1-\beta_{0}\right)}>0,
\end{aligned}
$$

which is positive as well for $E$ large enough.

For the remaining case $\left|\operatorname{Re} d_{0}\right| / 4 \geq\left|1+\operatorname{Im} d_{0}\right| \geq 0$, we see that $-\operatorname{Re}\left(\xi_{0}^{2}\right)>0$ due to (2.8) and (5.1). Hence we have

$$
\begin{aligned}
& \frac{R_{0}}{\pi^{2}\left|\xi_{0}\right|^{2}} \operatorname{Re} \frac{\mathrm{d} \rho\left(R_{0}\right)}{\mathrm{d} R} \\
\geq & 2 E\left\{|d+i|^{2}-\frac{\operatorname{Re}\left[\left(d_{0}+i\right)^{2} \xi_{0}^{2}\right]}{\left|\xi_{0}\right|^{2}}\right\}-\frac{a \beta_{0}}{\pi^{3} \beta_{1}\left(1-\beta_{0}\right)} \\
= & 2 E\left\{|d+i|^{2}-\frac{\left[\left(\operatorname{Re} d_{0}\right)^{2}-\left(1+\operatorname{Im} d_{0}\right)^{2}\right] \operatorname{Re}\left(\xi_{0}^{2}\right)-2\left(\operatorname{Re} d_{0}\right)\left(1+\operatorname{Im} d_{0}\right) \operatorname{Im}\left(\xi_{0}^{2}\right)}{\left|\xi_{0}\right|^{2}}\right\} \\
& -\frac{a \beta_{0}}{\pi^{3} \beta_{1}\left(1-\beta_{0}\right)} \\
\geq & 2 E\left[|d+i|^{2}+\frac{2\left(\operatorname{Re} d_{0}\right)\left(1+\operatorname{Im} d_{0}\right) \operatorname{Im}\left(\xi_{0}^{2}\right)}{\left|\xi_{0}\right|^{2}}\right]-\frac{a \beta_{0}}{\pi^{3} \beta_{1}\left(1-\beta_{0}\right)} \\
\geq & 2 E\left[|d+i|^{2}-\frac{1}{2}\left(\operatorname{Re} d_{0}\right)^{2}\right]-\frac{a \beta_{0}}{\pi^{3} \beta_{1}\left(1-\beta_{0}\right)} \\
\geq & E\left(\operatorname{Re} d_{0}\right)^{2}-\frac{a \beta_{0}}{\pi^{3} \beta_{1}\left(1-\beta_{0}\right)} .
\end{aligned}
$$

This also implies the desired assertion by letting $E$ be large enough. The proof of Theorem 5.1 is complete, subject to proving Lemma 5.1.

Proof. [Proof of Lemma 5.1] Let us first note, from (2.8) and Lemma 2.1, that

$$
\operatorname{Re} d_{1}\left|\eta_{1}\right|^{2}<-\operatorname{Re} d_{0}
$$

or, by (2.9),

$$
\frac{\beta_{0}\left(\beta_{1}-1\right)}{\beta_{1}\left(1-\beta_{0}\right)}>\left|d_{0}+i\right|^{2}=\left[\frac{2 \pi^{2} \beta_{0} \operatorname{Im} \rho-a \pi}{R_{0} a\left(1-\beta_{0}\right)}-1\right]^{2}+\left[\frac{2 \pi^{4} E \beta_{0}^{2}}{R_{0} a\left(1-\beta_{0}\right)}\right]^{2} .
$$


This implies

$$
\left|d_{1}\right|+\left|d_{2}\right|<c_{1},
$$

and $R_{0}>c_{2} E$ for some positive constants $c_{1}$ and $c_{2}$, independent of $E$ and $R_{0}$. Thus $E \rightarrow \infty$ implies $R_{0} \rightarrow \infty$.

On the contrary, we suppose that

$$
\liminf _{E \rightarrow \infty} \frac{E}{R_{0}}=0,
$$

which will lead to a contradiction. Indeed, by following the final step in the proof of Theorem 3.1, we use (2.9) or (3.9) to obtain

$$
\begin{aligned}
\lim _{E \rightarrow \infty} \gamma_{3} & =\frac{1}{-\frac{1}{\lim _{E \rightarrow \infty} i \operatorname{Im} d_{0}+i}-\lim _{E \rightarrow \infty} i \operatorname{Im} d_{1}}-\lim _{E \rightarrow \infty} i \operatorname{Im} d_{2} \\
& =i \frac{\lim _{E \rightarrow \infty} \operatorname{Im} d_{0}+1}{\lim _{E \rightarrow \infty} \operatorname{Im} d_{1}\left(\lim _{E \rightarrow \infty} \operatorname{Im} d_{0}+1\right)-1}-\lim _{E \rightarrow \infty} i \operatorname{Im} d_{2}
\end{aligned}
$$

and

$$
\lim _{E \rightarrow \infty}\left(\operatorname{Im} \gamma_{3}+\operatorname{Im} d_{2}\right)=\frac{\lim _{E \rightarrow \infty} \operatorname{Im} d_{0}+1}{\lim _{E \rightarrow \infty} \operatorname{Im} d_{1}\left(\operatorname{Im} d_{0}+1\right)-1},
$$

where, for simplicity of notation, we have supposed the existence of the limit.

Furthermore, multiplying (3.12) by $R_{0} / E$ and passing to the limit $E \rightarrow \infty$, we obtain, after applying (5.4), (5.5), (5.6), (5.7), (5.8) and the positivity of $\operatorname{Re} \gamma_{3}$,

$$
\begin{aligned}
\frac{R_{0}}{E}\left|\operatorname{Re} d_{0}\right|= & \frac{\lim _{E \rightarrow \infty} \frac{R_{0}}{E} \operatorname{Re}\left(d_{2}+\gamma_{3}\right)+\frac{R_{0}}{E} \operatorname{Re} d_{1} \lim _{E \rightarrow \infty}\left(\operatorname{Im} d_{2}+\operatorname{Im} \gamma_{3}\right)^{2}}{\left|\lim _{E \rightarrow \infty} \operatorname{Im} d_{1} \lim _{E \rightarrow \infty}\left(\operatorname{Im} d_{2}+\operatorname{Im} \gamma_{3}\right)-1\right|^{2}} \\
\geq & \frac{\frac{R_{0}}{E} \operatorname{Re} d_{2}+\frac{R_{0}}{E} \operatorname{Re} d_{1} \lim _{E \rightarrow \infty}\left(\operatorname{Im} d_{2}+\operatorname{Im} \gamma_{3}\right)^{2}}{\left|\lim _{E \rightarrow \infty} \operatorname{Im} d_{1} \lim _{E \rightarrow \infty}\left(\operatorname{Im} d_{2}+\operatorname{Im} \gamma_{3}\right)-1\right|^{2}}
\end{aligned}
$$

that is,

$$
\begin{aligned}
\frac{\beta_{0}^{2}}{1-\beta_{0}} \geq & \frac{\beta_{2}^{2}}{\beta_{2}-1}+\frac{\beta_{1}^{2}}{\lim _{1}-1} \lim _{E \rightarrow \infty}\left(\operatorname{Im} d_{1} \lim _{E \rightarrow \infty}\left(\operatorname{Im} d_{2}+\operatorname{Im} \gamma_{3}\right)^{2}\right)-\left.1\right|^{2} \\
& =\frac{\beta_{2}^{2}}{\beta_{2}-1}\left[1-\lim _{E \rightarrow \infty} \operatorname{Im} d_{1}\left(\operatorname{Im} d_{0}+1\right)\right]^{2}+\frac{\beta_{1}^{2}}{\beta_{1}-1}\left(\lim _{E \rightarrow \infty} \operatorname{Im} d_{0}+1\right)^{2} \\
& \geq \frac{\beta_{2}^{2}}{\beta_{2}-1}\left[1-\lim _{E \rightarrow \infty} \operatorname{Im} d_{1}\left(\operatorname{Im} d_{0}+1\right)\right]^{2} \\
& =\frac{\beta_{2}^{2}}{\beta_{2}-1}\left\{1+\lim _{R_{0} \rightarrow \infty} \frac{2 \pi^{2} \beta_{1} \operatorname{Im} \rho}{R_{0} a\left(\beta_{1}-1\right)}\left[\frac{2 \pi^{2} \beta_{0} \operatorname{Im} \rho}{R_{0} a\left(1-\beta_{0}\right)}-1\right]\right\}^{2} \\
& \geq \frac{\beta_{2}^{2}}{\beta_{2}-1}\left[1-\frac{\beta_{1}\left(1-\beta_{0}\right)}{4 \beta_{0}\left(\beta_{1}-1\right)}\right]^{2} .
\end{aligned}
$$


We thus have (3.13) or

$$
\frac{\left(a^{2}+1 / 4\right)^{2}}{3 / 4-a^{2}} \geq \frac{\left(a^{2}+25 / 4\right)^{2}}{a^{2}+21 / 4}\left[1-\frac{\left(a^{2}+9 / 4\right)\left(3 / 4-a^{2}\right)}{4\left(a^{2}+1 / 4\right)\left(a^{2}+5 / 4\right)}\right]^{2}>\frac{\left(a^{2}+1 / 4\right)^{2}}{3 / 4-a^{2}} .
$$

This leads to a contradiction, and hence (5.2) is valid. The proof of Lemma 5.1, and hence of Theorem 5.1, is complete.

As stated in Section 1, Assertion (i) of Theorem 1.1 was proven in Section 2, while Assertion (ii) follows by combining the results of Theorems 3.1, 4.1 and 5.1.

6. Symmetry Considerations. As discussed in Section 1, the symmetry properties of the domain and the forcing, on the one hand, and of the perturbation that gives rise to the bifurcation, on the other, have a decisive effect on whether the bifurcation leads to a branch of stationary or oscillatory solutions. Legras and Ghil [16] and Jin and Ghil [13] pointed out that, in the atmospheric channel problem with bottom topography, back-to-back saddle-node bifurcations resulted when the zonal forcing jet had a flat or unimodal velocity profile; see also Charney and DeVore [4] and Pedlosky [27]. To the contrary, when a higher-order component, which exhibited an inflection point, was present in the forcing jet, an oscillatory instability with so-called intraseasonal periods of 30-60 days could set in by resonance with this higher-order component, and lead to a stable limit cycle.

The periods of this type of instability are longer than the ones associated with the traveling Rossby waves that are the unique type of solutions of (1.1) in the same periodic-channel geometry and in the absence of forcing, topography, and dissipation. While all the numerical and asymptotic results cited herein used a periodic channel or spherical geometry with bottom topography, our rigorous result in Theorem 1.1 shows that Hopf bifurcation occurs even for a flat bottom of the channel. The separate effects of forcing and dissipation, on the one hand, and topography, on the other, on the period of oscillatory solutions will be studied further elsewhere.

On the other hand, in the oceanic rectangular-basin problem, given the antisymmetric wind-stress forcing profile of equation (1.1) here, a steady double-gyre circulation resulted that is antisymmetric with respect to the basin's zonal symmetry axis $[2,12,34]$. This circulation is first destabilized by a pitchfork bifurcation-perfect in QG models $[2,3,9]$ and perturbed in shallow-water models [12, 32, 33]) - that arises from a purely exponential instability, which is symmetric with respect to the symmetry axis defined here as $y=1$. Oscillatory instabilities documented numerically in either type of model, QG or shallow-water, also included some that have an asymmetric spatial pattern. The periods of these asymmetric instabilities are much longer than those of the oceanic problem's free modes (i.e., those arise in the absence of forcing and dissipation), the Rossby basin modes (see Simonnet and Dijkstra [31], and Simonnet et al. [33]).

The symmetry properties of the Hopf bifurcation here are thus in agreement with those obtained numerically for the atmospheric channel flow. The situation for the oceanic double-gyre problem is more complex and requires further investigation. It might be possible, using some of these symmetry ideas, to adapt the approach and methods used here to this oceanic problem, in spite of the fact that analytic stationary solutions are harder to find for it [36, 12].

Acknowledgement. It is a pleasure to thank Eric Simonnet for his insightful comments and suggestions on an earlier draft and for checking most of the inequalities. 


\section{REFERENCES}

[1] P. S. Berloff and S. P. Meacham, On the stability of the wind-driven circulation, J. Marine Res., 56 (1998), pp. 937-993.

[2] P. Cessi And G. R. IERley, Symmetry-breaking multiple equilibria in quasi-geostrophic, winddriven flows, J. Phys. Oceanogr., 25 (1995), pp. 1196-1202.

[3] K. -I. Chang, M. Ghil, K. Ide And C.-C. A. LaI, Transition to aperiodic variability in a wind-driven double-gyre circulation model, J. Phys. Oceanogr., 31 (2001), pp. 1260-1286.

[4] J. Charney and J. DeVore, Multiple flow equilibria in the atmosphere and blocking, J. Atmos. Sci., 36 (1979), pp. 1205-1216.

[5] Z. M. Chen And W. G. Price, Remarks on time-dependent periodic Navier-Stokes flow in a two-dimensional torus, Comm. Math. Phys., 207 (1999), pp. 81-106.

[6] Z. M. Chen And S. WANG, Steady-state bifurcations of the three-dimensional Kolmogorov problem, Electron. J. Differential Equations, 58 (2000), 1-32.

[7] H. A. Dijkstra, Nonlinear Physical Oceanography: A Dynamical Systems Approach to the Large-Scale Ocean Circulation and El Niño, Kluwer Acad. Publishers, Dordrecht/ Norwell, Mass., 2000, 456 pp.

[8] M. Ghil And S. Childress, Topics in Geophysical Fluid Dynamics: Atmospheric Dynamics, Dynamo Theory, and Climate Dynamics, Springer-Verlag, New York, 1987, 485pp.

[9] M. Ghil, Y. Feliks and L. Sushama, Baroclinic and barotropic aspects of the wind-driven ocean circulation, Physica D, 2002, to appear.

[10] D. Gilbarg and N. S. Trudinger, Elliptic Partial Differential Equations of Second Order, 2nd Edition, Springer-Verlag, New York, 1983.

[11] V. I. IUdovich, Example of the generation of a secondary stationary or periodic flow when there is loss of stability of the laminar flow of a viscous incompressible fluid, J. Math. Mech., 29 (1965), pp. 587-603.

[12] S. JiAng, F. -F. JiN AND M. GhIL, Multiple equilibria, periodic, and aperiodic solutions in a wind-driven, double-gyre, shallow-water model, J. Phys. Oceanogr., 25 (1995), pp. 764-786.

[13] F. -F. Jin AND M. GhiL, Intraseasonal oscillations in the extratropics: Hopf bifurcation and topographic instabilities, J. Atmos. Sci., 47 (1990), pp. 3007-3022.

[14] D. D. Joseph And D. Sattinger, Bifurcating time periodic solutions and their stability, Arch. Rational Mech. Anal., 45 (1972), pp. 75-109.

[15] E. Kalnay, Atmospheric Modeling, Data Assimilation and Predictability, Cambridge Univ. Press, Cambridge, MA, in press, 2002.

[16] B. Legras And M. Ghil, Persistent anomalies, blocking and variations in atmospheric predictability, J. Atmos. Sci., 42 (1985), pp. 433-471.

[17] J. L. Lions, R. Temam And S. WAng, New formulations of the primitive equations of atmosphere and applications, Nonlinearity, 5 (1992), pp 237-288.

[18] - On the equations of large-scale ocean, Nonlinearity, 5 (1992), pp. 1007-1053.

[19] E. N. Lorenz, Deterministic nonperiodic flow, J. Atmos. Sci., 20 (1963), pp. 130-141.

[20] - The mechanics of vacillation, J. Atmos. Sci., 20 (1963), pp. 448-464.

[21] _ The Nature and Theory of the General Circulation of the Atmosphere, WMO, Geneva, Switzerland, 1967, 161 pp.

[22] J. E. Marsden and M. McCracken, The Hopf Bifurcation and its Applications, SpringerVerlag, New York, 1976.

[23] S. P. Meacham, Low-frequency variability in the wind-driven circulation, J. Phys. Oceanogr., 30 (2000), pp. 269-293.

[24] S. P. MeAcham And P. S. Berloff, Instability of a steady, barotropic, wind-driven circulation, J. Marine Res. 55 (1997), pp. 885-913.

[25] L. D. Meshalkin and Ya. G. Sinai, Investigation of the stability of a stationary solution of a system of equations for the plane movement of an incompressible viscous fluid, J. Math. Mech., 19 (1961), pp. 1700-1705.

[26] B. Nadiga And B. Luce, Global bifurcation of Shilnikov type in a double gyre ocean model, J. Phys. Oceanogr., 31 (2001), pp. 2669-2690.

[27] J. Pedlosky, Resonant topographic waves in barotropic and baroclinic flows, J. Atmos. Sci., 38 (1981), pp. 2626-2641.

[28] — Geophysical Fluid Dynamics, 2nd Edition, Springer-Verlag, New-York, 1987.

[29] J. Shen, T. TAChim-Medjo And S. Wang, On a wind-driven, double-gyre, quasi-geostrophic ocean model: numerical simulations and structural analysis, J. Comp. Phys., 155 (1999), pp. 387-409.

[30] V. A. Sheremet, G. Ierley and V. Kamenkovich, Eigenanalysis of the two-dimensional wind-driven ocean circulation problem, J. Marine Res., 55 (1997), pp. 57-92. 
[31] E. Simonnet And H. Dijkstra, Spontaneous generation of low-frequency modes of variability in the wind-driven ocean circulation, J. Phys. Oceanogr., 2002, to appear.

[32] E. Simonnet, M. Ghil, K. Ide, R. Temam And S. Wang, Low-frequency variability in shallowwater models of the wind-driven ocean circulation. Part I: Steady-state solutions, J. Phys. Oceanogr., 2002, sub judice.

[33] _ Low-frequency variability in shallow-water models of the wind-driven ocean circulation. Part II: Time-dependent solutions, J. Phys. Oceanogr., 2002, sub judice.

[34] S. Speich, H. DiJKsTRA AND M. GhiL, Successive bifurcations in a shallow-water model, applied to the wind-driven ocean circulation, Nonlin. Proc. Geophys., 2 (1995), pp. 241-268.

[35] H. Stommel, Thermohaline convection with two stable regimes of flow, Tellus, 13 (1961), pp. 224-230.

[36] G. Veronis, An analysis of wind-driven ocean circulation with a limited number of Fourier components, J. Atmos. Sci., 20 (1963), pp. 577-593.

[37] - Wind-driven ocean circulation. Part II: Numerical solution of the nonlinear problem, Deep-Sea Res., 13 (1966), pp. 30-55.

[38] S. WANG, Attractors for the 3D baroclinic quasi-geostrophic equations of large-scale atmosphere, J. Math. Anal. Appl., 165 (1992), pp. 266-283.

[39] G. Wolansky, Existence, uniqueness, and stability of stationary barotropic flow with forcing and dissipation, Comm. Pure Appl. Math., 41 (1988), pp. 19-46.

[40] - The barotropic vorticity equation under forcing and dissipation: bifurcations of nonsymmetric responses and multiplicity of solutions, SIAM J. Appl. Math., 49 (1989), pp. $1585-1607$. 